\title{
Heart rate, energy expenditure, and affective responses from children participating in trampoline park sessions compared with traditional extra- curricular sports clubs \\ Post-print article published on: 5/2/2019 doi: $10.23736 /$ S0022-4707.18.09351-9
}

Research conducted by the ukactive Research Institute

Final Article In Press in Journal of Sports Medicine and Physical Fitness

Citation: Budzynski-Seymour, E, Wade, M, Lawson, R, Lucas, A, Steele, J. (2019). Heart rate, energy expenditure, and affective responses from children participating in trampoline park sessions compared with traditional extra-curricular sports clubs. Journal of Sports Medicine and Physical Fitness. DOI: 10.23736/S0022-4707.18.09351-9 


\section{SUMMARY OF KEY FINDINGS}

- Lack of physical activity is widely becoming a concern in younger populations. Inactivity has negative associations with quality of life, in addition to children's academic, social, and physical skills. Further, it may predispose them to poorer health later in life.

- For most children physical education and extracurricular activities delivered through school are the most apparent opportunities for engagement in physical activity.

- However, opportunities for physical activity exist outside of the school environment per se and a place that has seen an increase in popularity in recent years are trampoline parks.

- The emotional state (feelings) of a child during physical activity is thought to be related to their enjoyment and potentially adherence.

- Trampoline parks are typically thought of as being exciting adventure based activity and so it was of interest to examine both the heart rate and energy expenditure, in addition to affect, of children taking part in both extracurricular sports (football and netball) compared to a trampoline park session.

- A group of 26 children (aged 6-11 years, 16 girls and 10 boys) took part in the study. They wore heart rate belts during the activities and gave a rating of their feelings after them too. Data was collected from 3 sessions of both extracurricular sports in addition to trampoline park sessions.

- The results showed heart rate and energy expenditure were higher during trampoline park sessions, though both activities were classed as moderate-vigorous physical activity. Further, both activities elicited feelings of 'excitement'.

- Both extracurricular sports clubs and trampoline parks may be viable options for children to engage in physical activity that is sufficiently 'intense' and related to positive feelings. 


\title{
Heart rate, energy expenditure, and affective responses from children participating in trampoline park sessions compared with traditional extra- curricular sports clubs
}

\author{
Budzynski-Seymour, E. ${ }^{\mathrm{a}}$, Wade, M. ${ }^{\mathrm{b}}$, Lawson, R. ${ }^{\mathrm{b}}$, Lucas, A. ${ }^{\mathrm{b}}$, and Steele, J.a,
}

a'Solent University, Southampton, UK; bukactive Research Institute, London, UK; Contact for Correspondence emily.budzynski-seymour@solent.ac.uk; jamessteele@ukactive.org.uk; Twitter: @Emily_CBS; @ jamessteeleii

\begin{abstract}
SCIENTIFIC ABSTRACT
Background: Lack of physical activity (PA) is becoming an issue in younger populations. Trampoline parks are newly popular environments for PA yet research on their use is scarce. Thus the present study compared heart rate, energy expenditure, and affective responses in children participating in trampoline park sessions compared with extracurricular sports clubs. Methods: Children (aged 6-11 years; $n=16$ females, $n=10$ males) participated in 3 trampoline park sessions and 3 extracurricular sports club sessions lasting 45 minutes over 3 weeks. Heart rate, energy expenditure, and affective responses through the circumplex model, were measured. Results: Both conditions elicited moderate-vigorous PA. Average heart rate (mean difference [95\% CIs] $=27.6 f_{\mathrm{c}}[23.5$ to 31.8$]$ ), peak heart rate (mean difference [95\%CIs] $=24.2 f_{\mathrm{c}}[20.8$ to 27.6]) and energy expenditure (mean difference [95\%CIs] $=3.2 \mathrm{kcals} \mathrm{min}^{-1}$ [2.7 to 3.6]) were all significantly higher for the trampoline sessions. Affective responses for both conditions elicited feelings of 'excitement'. Conclusion: Both extracurricular sports clubs and trampoline park activities provide moderate-vigorous PA, though the latter may result in higher heart rate and energy expenditure responses. Both however produce similar positive affective responses. As such, both could be valuable options for PA opportunities for children.
\end{abstract}

Key words: physical activity; physical education; sports; youth

\section{INTRODUCTION}

Physical inactivity rates are increasing, with research into younger populations reporting both lower levels of physical activity (PA) and higher levels of sedentary behaviour, potentially contributing to childhood obesity which is now both a national and international issue. ${ }^{1,2}$ The poor health associated with low levels of PA has an adverse effect on the quality of life, and overall development of children's academic, social and physical skills. ${ }^{3}$ Furthermore, it may predispose them to poor health later on in life. ${ }^{3}$ Increasing levels of PA is likely to be key in averting childhood obesity and the preventable diseases that develop as a result. ${ }^{4}$ Indeed, the promotion of PA to improve the health of the younger population is a public health priority. ${ }^{2,5}$ There is increasing evidence that demonstrates the health enhancing effects of PA, and the detrimental effects of inactivity. Therefore, it is crucial researchers find ways to enhance young children's participation in PA. ${ }^{1,5}$

Assessing the physiological responses of PA engagement in children however, is a difficult task. There are a variety of methods currently available, yet heart rate monitors are a popular method due to their ease of measurement, ability to collect continuous data over a long period, and accurate representation of the stress that PA places on the cardiopulmonary system. ${ }^{6,7}$ Heart rate monitors are also useful due to the linear relationship that occurs between oxygen uptake and heart rate $^{7}$ and their use in measuring PA levels has been found to be both valid and reliable. ${ }^{6}$ In fact, they have been used to estimate energy expenditure in both young populations and adults, and when compared to whole body calorimetry, have shown acceptable validity. ${ }^{8}$

Although promotion of PA and its measurement is highlighted as a key priority for public health, it is important to 
remember that children differ from adults both in their motivations to take part in PA, and levels of PA required to maintain and improve health. Recent studies have called for a reform in children's exercise interventions as they are often given the same exercises as adults which may be inappropriate. ${ }^{1}$ Research shows that PA interventions in children are often unsuccessful resulting in the need for further studies to develop activities that can be used to increase the PA levels of children. ${ }^{4,9}$ Further, there is a growing interest in understanding the complex interrelationships between emotions, subjective physical feeling states, and PA in children. ${ }^{10}$

It has been suggested that a positive affective response during PA may lead to greater enjoyment of the exercise, thus playing a significant role in exercise adherence and indicating that affect may be the first link in the exercise adherence chain. ${ }^{9}$ Emotional states are key to the motivations behind behaviours including those which promote PA. ${ }^{10}$ It is possible that the reason behind many physical activity interventions being unsuccessful is the fact that they do not focus on the motivations behind engaging in PA. ${ }^{11}$ Research has found that children report enhanced positive affect after structured exercise tasks, and this suggests a bidirectional association between affective states and PA. ${ }^{10}$ However, it seems that certain PA types may lead to greater affective responses than others. For example, research has shown that structured gym activities (dodging, chasing, and fleeing games) resulted in more positive responses when compared to traditional aerobic activities (treadmill, cycle ergometer, and elliptical exercise ${ }^{1}$ ). Therefore, considering the affective response that PA may induce is crucial as a greater positive affect may lead to greater enjoyment and exercise adherence, which should increase PA levels and promote positive health outcomes. ${ }^{1}$
One location where children spend large amounts of time, and that can influence their PA levels, is at school during Physical Education (PE) lessons, and during extracurricular activities such as sports clubs. PE lessons have many important aims, such as the development of motor skills, creative and artistic expression, self-realisation, moral development and social development. ${ }^{12}$ However, although schools have the potential to be optimal venues for promoting PA levels, evidence shows that this is not the case, and many PA interventions at schools, including $\mathrm{PE}$ classes, do not have sufficiently positive impacts upon PA levels, ${ }^{5}$ or physical fitness. ${ }^{13}$ One reason behind these interventions being unsuccessful is the limitation of using curriculum time. The time allocated to PE lessons in schools is around 2 hours a week, which potentially limits the success of any intervention before it begins. ${ }^{14}$ One way of overcoming this issue is to utilise extracurricular time, such as after school activities as these will not impact educational time and provide an opportunity for facilitating PA engagement. ${ }^{14}$

As well as within school, children have many opportunities to engage in PA outside the school environment, with one such place being trampoline parks. Despite concerns regarding safety and injury risk, ${ }^{15}$ recently there has been a rise in the number of trampoline parks nationwide in the UK, which are opening at an exponential rate, and are perceived as an exciting adventure activity. ${ }^{16}$ It has been said that they help in the development of gross motor skills including jumping, balance and coordination, in addition to promoting the development of social skills and encouraging physical activity participation. ${ }^{16}$ A reason behind their popularity could be the need to identify environments where children can engage in PA, and enjoy doing so, thus potentially promoting regular PA participation. ${ }^{16}$ 
As trampoline parks have only recently gained a reputation as a place to engage in PA, research into the physical benefits of exercise at trampoline parks is scarce. However, due to their popularity, especially with the younger population, it seems prudent to investigate the benefits that they may bring. Therefore, the aim of this research was to investigate the heart rate response, estimated energy expenditure, and affective response from children participating in trampoline park sessions compared with traditional extracurricular sports clubs.

\section{METHODS \\ Study Design}

A repeated crossover study design was utilised whereby participants from a single school in the South of England participated in both trampoline park sessions, and traditional extracurricular sports clubs (soccer and netball), on three occasions. This design was used to compare between conditions heart rate responses, estimated energy expenditure and affective responses. Both soccer and netball were selected as it was anticipated that this would enable a greater number of both males and females to be recruited. The study design was approved by the Health, Exercise, and Sport Science Ethics Committee at the senior author's institution (ID: STEELE13032018).

\section{Participants}

An a priori sample estimate was performed using $\mathrm{G}^{*}$ Power (version 3.1.9.2; University of Kiel, Germany). This was based upon a moderate Cohen's $f$ of 0.25 at an $\alpha$ of 0.05 and $\beta$ of 0.80 and suggested 28 participants would be required. Recruitment was led by the school participating in the study. Letters were sent to parents indicating the study would be taking place and that children who were currently participating in extracurricular sports clubs including soccer and netball had the opportunity to opt-in through their current participation. Prior to participation, both children and their parents received participant information sheets with the recruitment letters, using language appropriate for children and parents. Both were offered the opportunity to ask any questions to the research team via email or telephone. If they were happy with the study outlined then both written informed consent was obtained from the parents, as well as written informed assent from the children. Twenty-nine children were recruited and participated in the study $(n=$ 17 females, $n=12$ males). After exclusion of participants who were recruited but did not complete at least one session of each condition, data was available for analysis from a total of 26 children ( $n=16$ females, $n=10$ males). Participants were aged $9 \pm 1$ years (range 6 to 11 years) with a height of $139.8 \pm 12.4 \mathrm{~cm}$, a weight of $35.5 \pm 10.1 \mathrm{~kg}$, and a body mass index of $18.0 \pm 3.6 \mathrm{~kg} . \mathrm{m}^{2}$.

\section{Equipment and Measures}

Heart rate was measured continuously throughout all sessions using chest worn heart rate monitors (MZ-3; Myzone Ltd, Isle of Man). The heart rate monitors were placed on the children by the research team prior to the sessions beginning, with the session start and end time noted to identify the correct time period from the heart rate data once exported. Children were instructed to alert a member of the research team if their heart rate monitor came loose and this was then adjusted for them to return to the session as quickly as possible. The heart rate belts measured heart rate every minute during the sessions and from this both average (AvgHR) and peak (PeakHR) heart rates were considered. From the heart rate data, energy expenditure in kcals was estimated using equations from Keytel et $\mathrm{al}^{17}$ and the mean rate of energy expenditure (kcal.min ${ }^{-}$ 1) across the session was calculated (AvgEE). Affective responses were also considered through the circumplex model. ${ }^{18}$ Valence was measured using the Children's Feeling Scale (CFS) and activation measured using the Children's

Page 4 Post-print article published on 5/2/2019 (doi: 10.23736/S0022-4707.18.09351-9) - The authors confirm they are happy to share this work. 
Felt Arousal Scale (CFAS) which were adapted from the adult versions by Hulley et al. ${ }^{19}$ When the sessions ended the participants returned to the researchers to have their heart rate monitors removed, and were then asked to provide ratings for both the CFS and CFAS. The children were shown the scales and asked "How do you feel right now?" and "How awake do you feel right now?" and to point to the number they felt most appropriate. During the first testing session the scales were described to the children and they were told to consider the faces as well to help them determine the most appropriate number.

\section{Trampoline Park Sessions \& Extracurricular Sport Clubs (Soccer and Netball)}

Both the trampoline park sessions and extracurricular sports clubs lasted for $\sim 45$ minutes and took place between $3 \mathrm{pm}$ and $5 \mathrm{pm}$ after school on weekdays. Sessions took place over a four-week period in May with the first sessions taking place in week 1 , and the second and third sessions taking place in week three and four respectively. This was due to data collection falling on a week with a national holiday in week two. Trampoline sessions took place on Mondays, and the extracurricular sport clubs on Thursdays (soccer) and Fridays (netball). Both sessions were supervised and delivered by in situ staff at the trampoline park or the school dependent upon the sessions.

Prior to beginning the trampoline park sessions all children were required to watch a safety video explaining the rules of the park. After this the session began and followed a similar format for each of the three sessions. The aim was to provide a fun, active experience for the children utilising a range of different activity types. This included an initial warmup followed by games of dodgeball, tag, obstacle courses, coaching of trampoline skills, and free time.

Extracurricular sports club sessions participants completing one or the other based upon which club they attended upon recruitment. These clubs took place at the participants' school and were delivered by trained PE and/or external (for soccer) staff. Both sessions followed similar formats, with children first performing a warmup followed by skills based activities (e.g. passing, defending, movement and support) incorporated into small games, finished up with a traditional match based activity.

\section{Statistical Analysis}

Due to the hierarchical structure of the data multilevel mixed modelling was used to account for clustering at the participant level. The hierarchical structure of the data included participants as a level 3 variable, condition as a level 2 , and time as a level 1. The effects of 'condition' (trampoline park sessions compared or extracurricular sports clubs), 'time' (session 1, 2, or 3), and 'condition $\mathrm{x}$ time' upon the dependent variables of AvgHR, PeakHR, AvgEE, CFS, and CFAS were examined. Fixed factors included in the model were 'condition', 'time', and 'condition x time', with 'sex' included as a fixed covariate and the 'condition $\mathrm{x}$ sex' interaction included to account for the discrepant sex distribution between the soccer and netball sessions within the extracurricular sports clubs condition. Random intercepts by participant were included, and random slopes for 'condition' and 'time' using an unstructured covariance type. Due to the inclusion of both fixed and random effects Restricted Maximum Likelihood estimation was used. Estimated marginal means and $95 \%$ confidence intervals were calculated and presented with comparisons made using post hoc Bonferonni tests. Analyses were performed using SPSS (version 23; IBM, Portsmouth, Hampshire, UK) with statistical significance accepted at $\alpha=0.05$. included both soccer and netball, with Page 5 Post-print article published on 5/2/2019 (doi: 1 10.23736/S0022-4707.18.09351-9) - The authors confirm they are happy to share this work. 


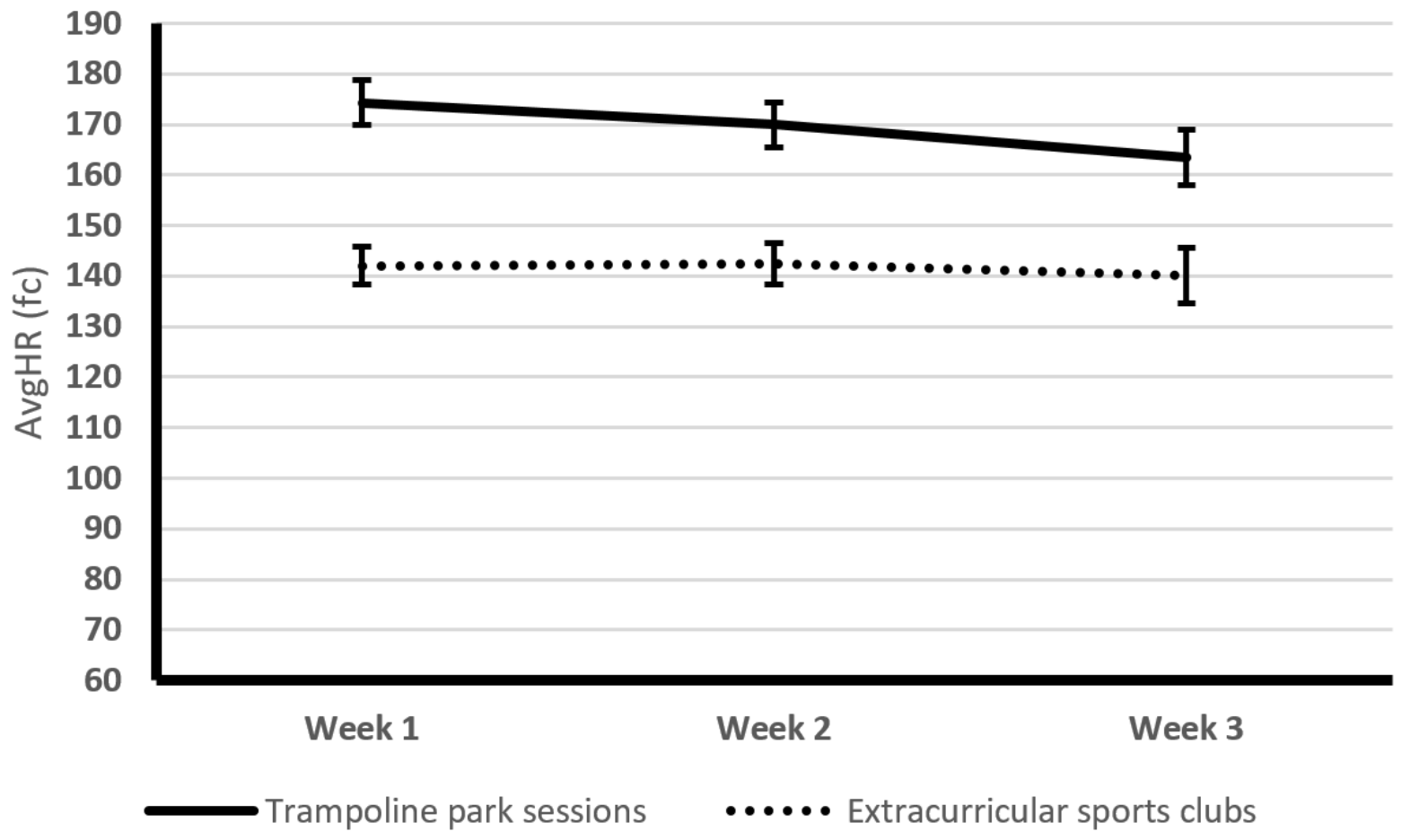

Figure 1. AvgHR responses for each condition and week. Data are presented as estimated marginal means $\pm 95 \%$ CIs.

\section{RESULTS}

\section{Heart Rate Responses}

For AvgHR there were significant main effects by 'condition' $\left(F_{(19.469)}\right)=$ $46.930, p \leq 0.001)$, 'time' $\left(F_{(13.430)}=6.623\right.$, $p=0.010)$, 'condition $\mathrm{x}$ time' $\left(F_{(76.476)}=\right.$ $4.468, p=0.015)$, and 'condition $\mathrm{x}$ sex' $\left(F_{(20.435)}=6.584, p=0.006\right)$. Pairwise comparisons of estimated marginal means for 'condition' revealed significantly greater AvgHR for the trampoline park sessions compared to the extracurricular sports clubs (mean difference $[95 \% \mathrm{CIs}]=$ 27.6 [23.5 to 31.8], $p \leq 0.001)$. For 'time' pairwise comparisons revealed no significant differences between week 1 and week 2 (mean difference [95\% CIs] $=2.0$ [2.4 to 6.3 ], $p=0.752$ ), or week 2 and week 3 (mean difference [95\% CIs] $=4.3[-0.2$ to
8.9], $p=0.064)$; however, there was a significant difference between week 1 and week 3 (mean difference [95\%CIs] $=6.3$ [0.8 to 11.8], $p=0.027$ ). Pairwise comparisons of 'condition $\mathrm{x}$ time' revealed no differences between any weeks for extracurricular sports clubs (all $p \geq 0.999$ ), and for trampoline park sessions there were no significant differences between week 1 and week 2 (mean difference [95\%CIs] = 4.4 [-1.1 to 9.9], $p=0.159$ ). However, there were significant differences between week 1 and week 3 (mean difference [95\%CIs] = 10.8 [4.6 to 17.0], $p=0.001$ ), and week 2 and week 3 (mean difference [95\% CIs] = 6.4 [0.8 to 11.9], $p=0.019$ ). AvgHR is shown in 1 for each condition and week.

For PeakHR there were significant main effects by 'condition' $\left(F_{(20.048)},=\right.$ 


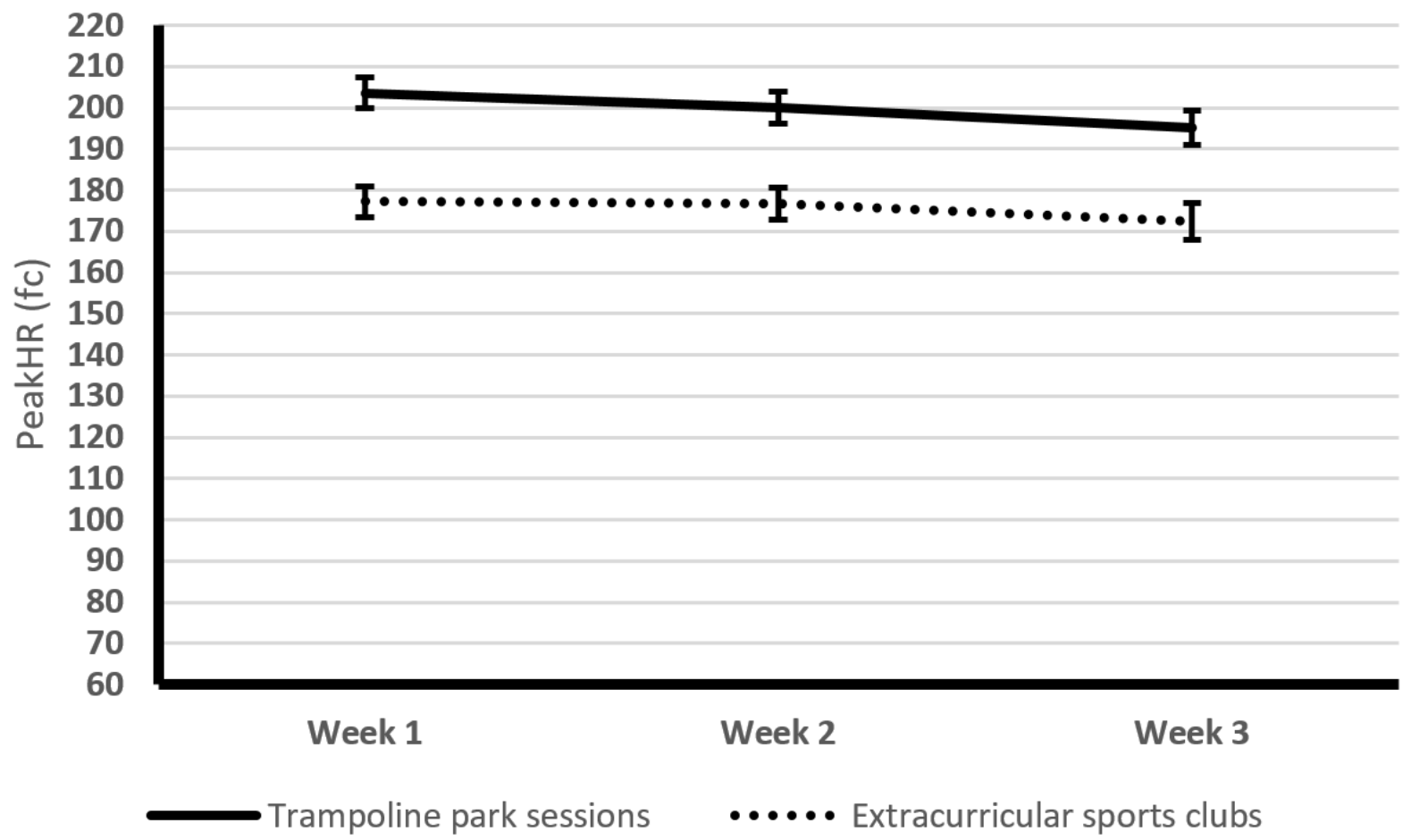

Figure 2. PeakHR responses for each condition and week. Data are presented as estimated marginal means $\pm 95 \%$ CIs.

63.247, $p \leq 0.001)$, 'time' $\left(F_{(18.364)}=\right.$ $10.591, p \leq 0.001)$, and 'condition $\mathrm{x}$ sex' $\left(F_{(21.167)}=10.641, p=0.001\right)$, but not 'condition $\mathrm{x}$ time' $\left(F_{(65.148)}=1.027, p=\right.$ 0.364). Pairwise comparisons of estimated marginal means for 'condition' revealed significantly greater PeakHR for the trampoline park sessions compared to the extracurricular sports clubs (mean difference $[95 \% \mathrm{CIs}]=24.2$ [20.8 to 27.6], $p \leq 0.001)$. For 'time' pairwise comparisons revealed no significant differences between week 1 and week 2 (mean difference $[95 \% \mathrm{CIs}]=0.3[-0.3$ to $0.9], p=0.634$ ), or week 2 and week 3 (mean difference $[95 \% \mathrm{CIs}]=4.3[-0.2$ to 8.9], $p=0.064)$; however, there was a significant difference between week 1 and week 3 (mean difference [95\%CIs] $=6.3$ [0.8 to 11.8], $p=0.027$ ). Pairwise comparisons of 'condition $\mathrm{x}$ time' revealed no differences for extracurricular sports clubs between week 1 and week 2 (mean difference $[95 \% \mathrm{CIs}]=0.6[-4.1$ to 5.3$], p \geq$ 0.999), week 1 and week 3 (mean difference $[95 \% \mathrm{CIs}]=4.9[-0.1$ to 9.9$], p=$ 0.058), or week 2 and week 3 (mean difference $[95 \% \mathrm{CIs}]=4.3[-1.1$ to 9.7$], p=$ 0.167). For trampoline park sessions there were no significant differences between week 1 and week 2 (mean difference [95\%CIs] $=3.6$ [ -1.3 to 8.5$], p=0.232$ ), or week 2 and week 3 (mean difference [95\%CIs] $=4.8$ [-0.4 to 10.0$], p=0.075)$; however, there was a significant difference between week 1 and week 3 (mean difference [95\%CIs] $=8.4$ [3.4 to 13.4], $p \leq$ $0.001)$. PeakHR is shown in figure 2 for each condition and week.

\section{Energy Expenditure}

For AvgEE there were significant main effects by 'condition' $\left(F_{(14.654)}\right.$, $=$ $20.561, p \leq 0.001)$, 'time' $\left(F_{(14.357)}=6.684\right.$, $p=0.009)$, and 'condition $\mathrm{x}$ sex' $\left(F_{(16.617)}=\right.$ 16.617, $p \leq 0.001)$, but not 'condition $\mathrm{x}$ time' $\left(F_{(83.009)}=1.754, p=0.179\right)$. Pairwise comparisons of estimated marginal means for 'condition' revealed significantly greater AvgEE for the trampoline park sessions compared to the extracurricular sports clubs (mean difference [95\%CIs] = 3.2 [2.7 to 3.6], $p \leq 0.001$ ). For 'time' pairwise comparisons revealed no significant differences between week 1 and 


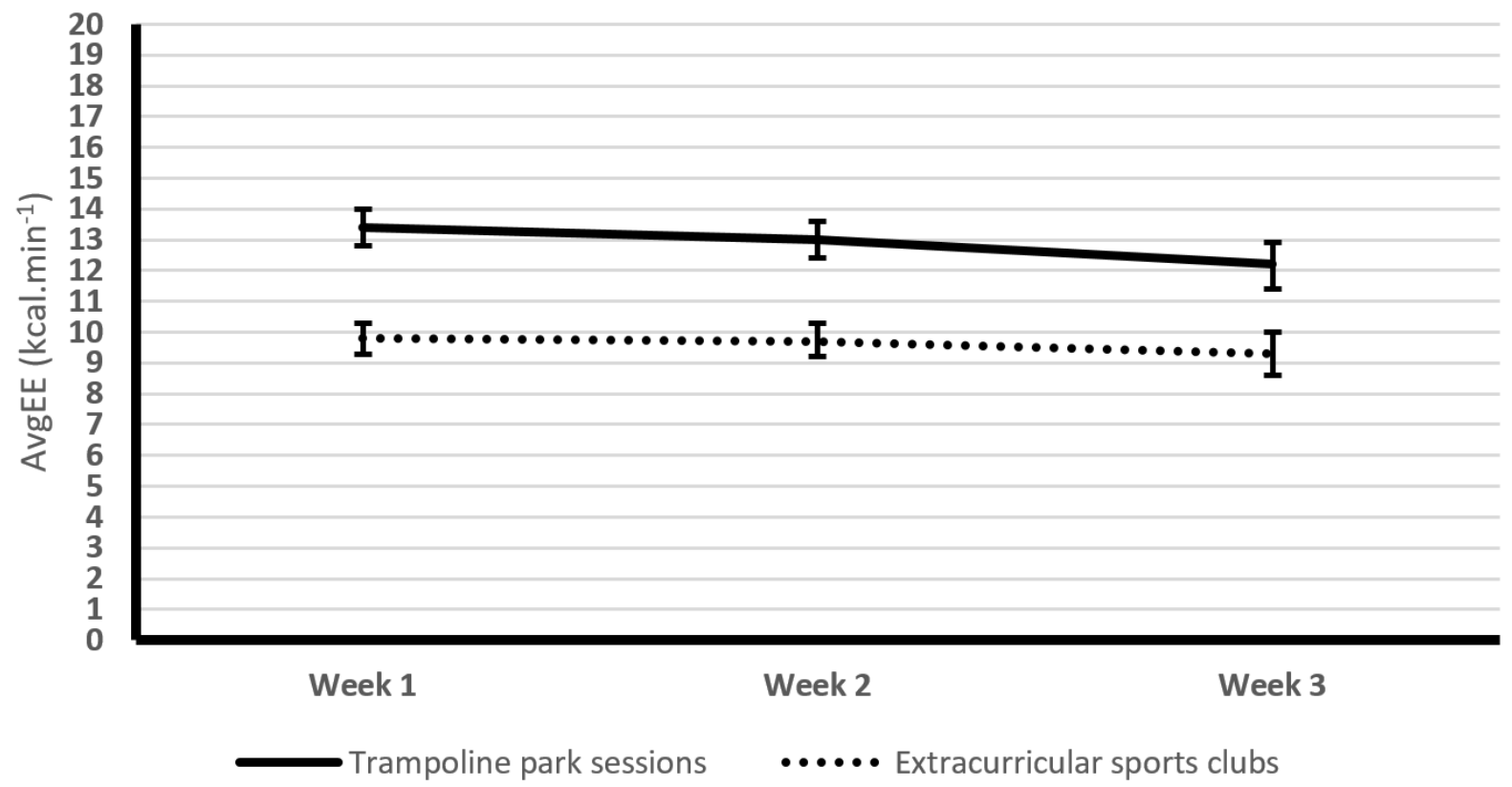

Figure 3. AvgEE responses for each condition and week. Data are presented as estimated marginal means $\pm 95 \%$ CIs.

week 2 (mean difference [95\%CIs] $=0.3[-$ 0.3 to 0.9 ], $p=0.634$ ), or week 2 and week 3 (mean difference [95\% CIs] $=0.6[-0.0$ to $1.2], p=0.062)$; however, there was a significant difference between week 1 and week 3 (mean difference [95\%CIs] $=0.9$ [0.1 to 1.6], $p=0.022$ ). Pairwise comparisons of 'condition $\mathrm{x}$ time' revealed no differences for extracurricular sports clubs between week 1 and week 2 (mean difference $[95 \% \mathrm{CIs}]=0.1[-0.6$ to 0.8$], p \geq$ 0.999), week 1 and week 3 (mean difference $[95 \% \mathrm{CIs}]=0.5[-0.4$ to 1.4$], p=$ 0.409 ), or week 2 and week 3 (mean difference [95\%CIs] $=0.4$ [-0.4 to 1.2$], p=$ $0.704)$. For trampoline park sessions there were no significant differences between week 1 and week 2 (mean difference [95\%CIs] $=3.6[-1.3$ to 8.5$], p=0.232$ ); however, there were significant differences between week 1 and week 3 (mean difference [95\% CIs] $=1.3$ [0.4 to 2.1], $p=$ 0.003 ), and week 2 and week 3 (mean difference $[95 \% \mathrm{CIs}]=0.8[-0.1$ to 1.6$], p=$ 0.034). AvgEE is shown in figure 3 for each condition and week.

\section{Affective Responses}

For CFS there were significant main effects by 'condition' $\left(F_{(16.475)},=4.795, p=\right.$ $0.043)$, but not 'time' $\left(F_{(13.936)}=1.479, p=\right.$ $0.262)$, 'condition $\mathrm{x}$ time' $\left(F_{(76.279)}=0.046\right.$, $p=0.955)$, or 'condition $\mathrm{x}$ sex' $\left(F_{(19.012)}=\right.$ $2.534, p=0.106)$. Pairwise comparisons of estimated marginal means for 'condition' revealed significantly greater CFS for the extracurricular sports clubs compared to the trampoline park sessions (mean difference [95\%CIs] $=0.8$ [0.1 to 1.5$], p=0.021)$.

For CFAS there were significant effects by 'condition' $\left(F_{(12.911)},=10.790, p\right.$ $=0.006)$ and 'condition $\mathrm{x}$ sex' $\left(F_{(6.485)},=\right.$ $5.488, p=0.040)$, but not 'time' $\left(F_{(23.794)},=\right.$ $2.466, p=0.106)$ or 'condition $\mathrm{x}$ time' $\left(F_{(89.081)},=1.006, p=0.370\right)$. However, pairwise comparisons of estimated marginal means for 'condition' however revealed no significant difference for CFAS between the extracurricular sports clubs compared to the trampoline park sessions (mean difference $[95 \% \mathrm{CIs}]=0.4[-0.1$ to $0.8], p=0.104)$. 


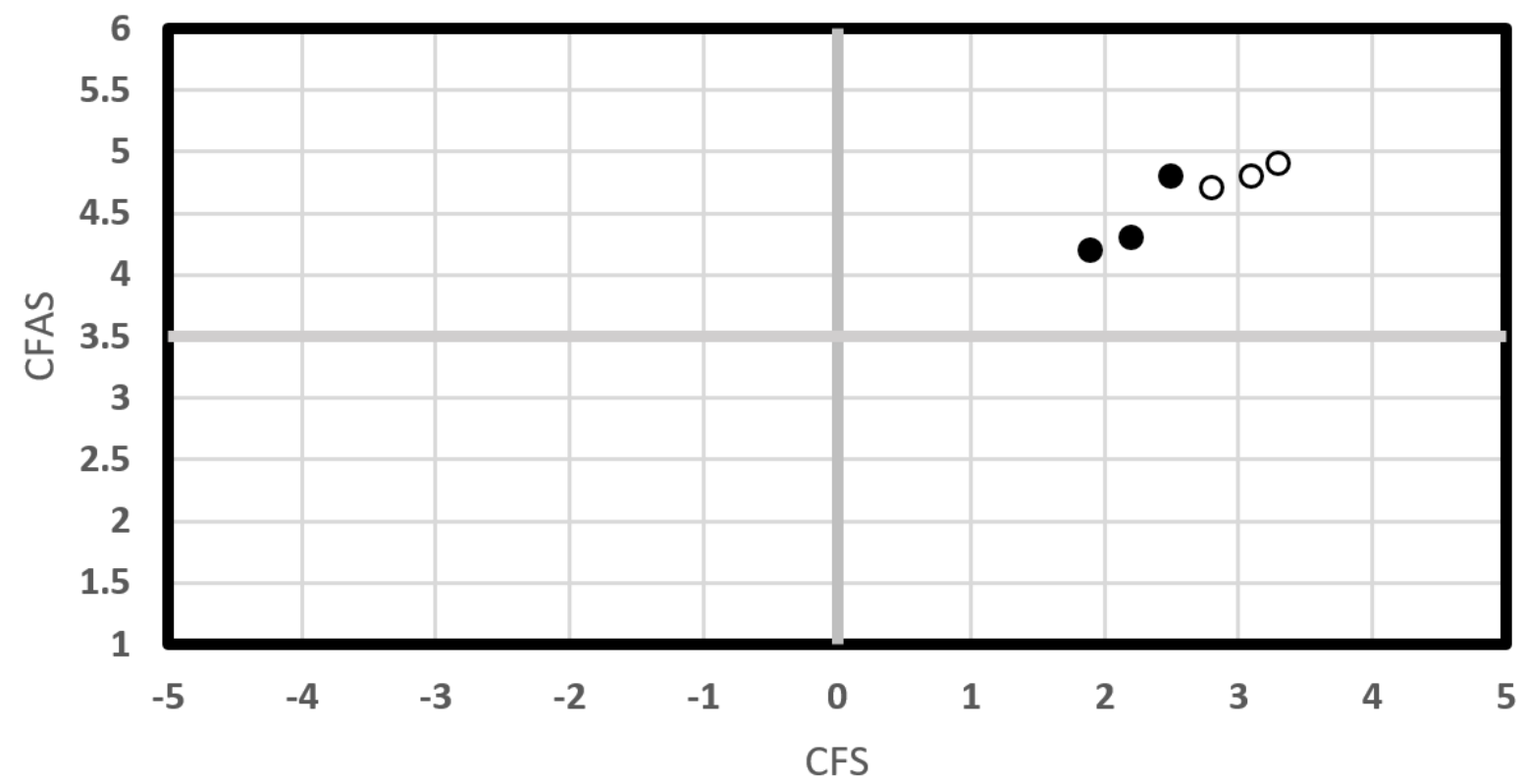

Figure 4. CFS and CFAS plotted in circumplex space.

CFS and CFAS were considered through the circumplex model of affect. A quadrant plot is shown in Figure 4 which suggested that, despite statistically significant differences between conditions for CFS and CFAS, these were small and both conditions elicited feelings of energy/enthusiasm.

\section{DISCUSSION}

The aim of this research was to investigate the heart rate response, estimated energy expenditure, and affective response from children participating in trampoline park sessions compared to traditional extracurricular sports clubs. The results showed that for AvgHR, PeakHR, and AvgEE, though both conditions elicited relatively high responses, these were significantly greater for the trampoline based exercise when compared to the extracurricular activities $(p \leq 0.001)$. For the affective response, the CFS results were significantly greater for the extracurricular activities $(p=0.021)$, but no significant differences were found for the CFAS scores $(p=0.104)$. However, when considered in the circumplex model both conditions appeared to elicit similar responses. Further, no adverse events (e.g. injuries) occurred during any of the sessions completed or were reported afterwards as having resulted from the sessions.

The results for both AvgHR and PeakHR were both significantly higher for the trampoline park sessions suggesting that the children experienced greater physiological stress, which may lead to greater adaptation of the cardiovascular system $^{7,20}$ when compared to extracurricular activities. Both high AvgHR and PeakHR is indicative of greater intensities of effort, and children aged between 5-17 are recommended to complete at least 60 minutes of moderate to vigorous intensity physical activity daily. ${ }^{21}$ Using the average age of the sample (9 years), to be exercising at a moderate to vigorous intensity the heart rate values would need to be between 135 to $206 f_{\mathrm{c}}{ }^{22}$ The results from the current study show that both the trampoline exercise and extracurricular activities meet these recommendations, though the trampoline exercise may be slightly superior as it yields higher values pushing the PA into the vigorous band. 
Extracurricular activities including soccer and netball have been investigated previously in the literature. Szakaly et al ${ }^{20}$ analysed primary school children's heart rate when performing a variety of physical activities; the average heart rate values for children ages 10-12 performing ball games was $156 f_{\mathrm{c}}$. This value is slightly higher than that recorded for the present study; however, this may be explained by participants in our study being slightly older. Szakaly et $\mathrm{al}^{20}$ also found that the activity with the highest heart rate values was "games", with average heart rates recorded as $164 f_{\mathrm{c}}$. Interestingly, this only reaches the lower range of the average AvgHR for all three weeks of the trampoline sessions which ranged from 164-175 $f_{\mathrm{c}}$. This was also the case in a similar study by Bendiksen et $\mathrm{al}^{23}$ who investigated the heart rate response of 8-9 year olds in various physical activities. The sample here were closer to the average age of the current sample ( $\sim 9$ years), with the highest average heart rate recorded for any activity as $156 f_{\mathrm{c}}$ which was for soccer, followed by basketball at $155 f_{\mathrm{c}}$ and unihockey at $152 f_{\mathrm{c}}$. Both of these studies show that the AvgHR values for young children participating in activities such as soccer are similar to the results shown in the current study; however, these are significantly lower than the AvgHR values reported for the trampoline sessions, suggesting a potential advantage over more traditional extracurricular activities. This suggests that the trampoline sessions may result in greater stimulus to the cardiovascular system and subsequently the overall health of the child.

Indeed, a recent study ${ }^{13}$ compared recreational soccer, traditional PE classes, and 'High Intensity Interval Training' (HIIT) in overweight and obese males aged 11-13 years. Average heart rates in this study were $75.1 \pm 2.3 \%, 68.3 \pm 2.2 \%$, and $80.0 \pm 3.0 \%$ of max heart rate for soccer, traditional PE classes, and HIIT respectively. In the present study the AvgHR was $77.2 \%$ to $82.5 \%$ for the Page | 10 trampoline classes and $67.2 \%$ to $67.5 \%$ for the traditional extracurricular classes. Thus comparatively, the extracurricular classes in the present study were more similar to traditional PE in the study of Cvetkovic et al. $^{13}$ Indeed, Cvetkovic et $\mathrm{al}^{13}$ also examined improvements in muscular and cardiorespiratory fitness over a 12-week intervention and found that the recreational soccer and HIIT intervention improved these outcomes suggesting mode of PA may be unimportant, yet the traditional PE classes resulted in increased body mass, BMI, and fat mass. This may be due to the relatively lower intensity of effort and thus stimulus provided by these classes as this is likely more important for adaptation as opposed to both modality and even PA bout duration. ${ }^{24,25}$ However, the estimated average caloric expenditure was also relatively less in the PE sessions compared with the soccer and HIIT sessions and thus may explain the body composition results.

An increased energy expenditure helps to combat excess weight gain in school children. ${ }^{26}$ The results of the current study suggest that energy expenditure is significantly greater $(p \leq 0.001)$ when engaging in a trampoline session compared to extracurricular sports clubs. The energy expenditure ranged from 12.1 to 13.2 kcal.min ${ }^{-1}$ for the trampoline session, and 9.5 to $9.8 \mathrm{kcal}^{\mathrm{min}} \mathrm{m}^{-1}$ for the extracurricular activities. Thus for 45 minute sessions the total estimated energy expenditure for the trampoline sessions were 544 to 594 kcals, and for the extracurricular sessions 428 to 441 which, despite the heart rate results, were actually both higher than the energy expenditures reported by Cvetkovic et $\mathrm{al}^{13}$ for soccer, HIIT, and PE classes.

There are several ways of considering energy expenditure, a common one being metabolic equivalents (METS). This is defined as the ratio of work metabolic rate to a standard metabolic rate, usually resting, and can range from as low as 0.9 (sleeping) to as high as 18 (running at $10.9 \mathrm{mph}$ ) with many activities listed in the Compendium of Physical Activities. ${ }^{27}$ 
When considering METS from the Compendium of Physical Activities, soccer is estimated as 6 METS and above, netball between 3.0-5.99 METS and trampoline exercise 3.5 - 4.5 METS (Ainsworth et al., 2000). These estimates however are specific to adults and thus recently, due to the relatively greater energy expenditure in children, ${ }^{28}$ a Youth Compendium of Physical Activities has been produced. ${ }^{29}$ From this and for the age groups included, soccer based activities range from 5.1 to 8.5 METS and trampoline sessions range from 7.3 to 8.2 METS (netball is not available). Converting the kcal.min ${ }^{-1}$ energy expenditure estimates in the present study to METS (using the average body mass for the group) for comparison shows 19.8 to 21.6 METS for the trampoline session, and 15.5 to 16.0 for the extracurricular activities. It is not clear as to why energy expenditures were so high comparative to those reported in the Compendium. Differences between the reported trampoline sessions and our estimates may be due to the nature of that activity specifically, such as traditional trampoline based use, rather than at an indoor activity center specifically designed for this. The notion of a "trampoline park" is new and therefore comparison with the more traditional trampoline scores may not be appropriate. Both activities though appear to elicit relatively higher energy expenditures than typically thought. Thus both might be options for the consideration of physical activity interventions for children with the aim of preventing weight gain.

Engagement in and adherence to physical activity is however thought to be impacted by the affective response to that physical activity. The affective responses to any context can be considered from two primary dimensions of the circumplex model: valence and activation, measured in this study by the CFS and CFAS respectively. Both trampoline sessions and extracurricular sports clubs elicited feelings of energy and enthusiasm, suggesting that Page | 11 both PA modes induce positive mental states in those participating. This is key as evidence shows that a greater positive affect experienced during PA leads to greater enjoyment of the session, resulting in a positive memory of the time spent engaging in PA and thus promotes exercise adherence. ${ }^{1}$ Indeed, this can result in a cycle whereby positive affective responses to PA results in further PA which further results in positive affective responses. ${ }^{10}$ Ensuring a positive affective response is pivotal to improving PA and the health of children; therefore it is important to promote those physical activities that lead to this.

As noted, in the current study both the trampoline based exercise and the extracurricular activities had this positive response, but this is not a consistent theme in the literature. Allender et $\mathrm{al}^{30}$ examined participation in sport and PA and argued that young children found exercise more enjoyable when they were not being forced to compete and win, but instead were encouraged to experiment with various activities. Preferences also impact upon PA choices in younger populations in addition to whether the activity is perceived to be 'fun'. ${ }^{31}$ Indeed, as noted, White et $\mathrm{al}^{1}$ reported game based gym activities to result in more positive affective response compared to traditional aerobic exercises. However, it may be that the two activity modes examined in the present study elicited similar responses for slightly different reasons. The participants were already participating in extracurricular sports clubs (soccer or netball), which could have resulted in an element of sampling bias; these activities were selfselected and thus likely to be perceived as 'fun'. The trampoline session however (though some children reported having attended the location previously due to proximity in location) may have provided more novelty and opportunity for experimentation. Either way, it could be argued that both should be considered and offered to children as opportunities for PA 
to enable them to self-select that which they prefer.

It should be noted that we also did not experience any adverse events such as injuries in the present study. Recent work has suggested that trampoline park related injuries are on the rise. ${ }^{15}$ However, this is likely a function of the growing popularity of these venues as places to engage in this activity and thus potentially a function of increased exposure. Further, proportionately only $11 \%$ of all trampoline injuries in the study of Kasmire et $\mathrm{al}^{15}$ occurred at trampoline parks and instead the majority occurred at home. Of course any activity has a potential risk of injury of varying severity and this is no different for trampoline park sessions, or indeed extracurricular sports clubs. In fact, differences in accidental injury rates differ little between different sports categories in children. ${ }^{32}$ Further, though of course trampoline activities can result in severe injuries it has been argued that there is little evidence to support a ban and instead the use of, and adherence to, appropriate safety guidelines is important ${ }^{33}$ which as noted was the case in the present study.

\section{Limitations and future research}

A key limitation of this study was the lack of random order to the participation in the conditions. Due to logistic constraints we were unable to randomize this. Had we done so we might have been able to consider the study as a replicated cross-over design enabling us to conduct analysis for identification of true inter-individual response variation ${ }^{34}$ to understand whether certain children had better responses to certain conditions. A further limitation is that the participants were children already self-selected to engage in PA as they were already participating in extracurricular sports clubs. It may be that the results, in particular the heart rate and energy expenditures, may differ with children who were not inclined to participate in such activities. It is perhaps also likely that the affective responses might differ if children Page | 12 who had not chosen to take part (i.e. if it had been delivered as part of core PE classes) were included instead. Lastly, the final sample size was slightly lower than that estimated $a$ priori (26 vs 28 participants); however, this seems to have had little impact upon the findings.

\section{CONCLUSION}

This study supports previous research into PA and illustrates both acute physiological and affective benefits of engaging in PA. Although both extracurricular sports club activities (soccer and netball) and the trampoline park sessions were considered to be moderatevigorous $\mathrm{PA}$, the latter may provide greater and more significant responses for a heart rate and energy expenditure suggesting it to be more vigorous. However, both activities led to similarly positive affective responses suggesting that both might be activities that children will perceive positively and thus potentially engage in. As such, we suggest that children should be offered a range of activity opportunities including those examined here. Future research however might look to examine trampolines parks compared with other traditional PE class activities in more representative samples (i.e. including children who have not selfselected to comparator activity.

\section{ACKNOWLEDGMENTS}

We would like to thank Myzone for providing us with access to the heart rate monitors in order to complete the data collection. We would also like to thank the staff and pupils at Thornhill Primary School in Southampton, UK in addition to the staff at Oxygen Freejumping who facilitated the delivery of the extracurricular sessions and trampoline sessions respectively.

\section{AUTHOR CONTRIBUTIONS}

EBS contributed to the data collection, data analysis and interpretation, and drafted the manuscript; MW, AL, and RL contributed to the study design, data interpretation, and critical revision of the 
manuscript; JS contributed to the study design, data collection, data analysis and interpretation, and drafted the manuscript

\section{FUNDING SOURCE}

This research was funded by Oxygen Freejumping who also provided access to their centre in Thornhill, Southampton, UK for completion of data collection. Other than the available times for access to the centre for this purpose which impacted on the ability to randomise order of the testing sessions, Oxygen Freejumping had no other role in the study design, data collection, analysis, or interpretation, the writing of this report, or in the decision to submit the article for publication.

\section{REFERENCES}

1. White DA, Rothenberger SD, Hunt LA, Goss FL. Comparison of Affect and Cardiorespiratory Training Responses Between Structured Gym Activities and Traditional Aerobic Exercise in Children. Int J Exerc Sci. 2016; 9(1): 16.

2. Lee JE, Stodden DF, Gao Z. Young children's energy expenditure and moderate-to-vigorous physical activity on weekdays and weekends. J Phys Act Health. 2016; 13(9): 1013-1016.

3. Domone S, Mann S, Sandercock G, Wade M, Beedie C. A method by which to assess the scalability of field-based fitness tests of cardiorespiratory fitness among schoolchildren. Sports Med. 2016; 46(12): 1819-1831.

4. Duncan MJ, Clarke ND, Birch SL, Tallis J, Hankey J, Bryant E, Eyre EL. The effect of green exercise on blood pressure, heart rate and mood state in primary school children. Int J Environ Res Public Health. 2014; 11(4): 3678-3688.

5. Schneider M, Cooper DM. Enjoyment of exercise moderates the impact of a school-based physical activity intervention. Int $\mathrm{J}$ Behav Nutr Phys Act. 2011; 8(1), 64.

6. Armstrong N. Young people's physical activity patterns as assessed by heart rate monitoring. J Sports Sci. 1998; 16(sup1): 9-16.

7. Eston RG, Rowlands AV, Ingledew DK. Validity of heart rate, pedometry, and accelerometry for predicting the energy cost of children's activities. J Appl Physiol. 1998; 84(1), 362-371.

8. Bitar A, Vermorel M, Fellmann N, Bedu M, Chamoux A, Coudert J. Heart rate recording method validated by whole body indirect calorimetry in 10-yr-old children. J Appl Physiol. 1996; 81(3): 11691173.

9. Benjamin CC, Rowlands A, Parfitt G. Patterning of affective responses during a graded exercise test in children and adolescents. Pediatr Exerc Sci. 2012; 24(2): 275-288.

10. Dunton GF, Huh J, Leventhal AM, Riggs N, Hedeker D, Spruijt-Metz D, Pentz MA. Momentary assessment of affect, physical feeling states, and physical activity in children. Health Psychol. 2014; 33(3): 255-263.

11. Carpenter J G, Bélanger M, O'Loughlin J, Xhignesse M, Ward S, Caissie I, Sabiston C. Association between physical activity motives and type of physical activity in children. Intl J Sport Exerc Psychol. 2017; 15(3): 306-320.

12. Stratton G. Children's heart rates during British physical education lessons. J Teach Phys Ed. 1997; 16(3): 357-367.

13. Cvetković N, Stojanović E, Stojiljković N, Nikolić D, Scanlan AT, Milanović Z. Exercise training in overweight and obese children: Recreational football and highintensity interval training provide similar benefits to physical fitness. 
Scand J Med Sci Sports. 2018; Epub ahead of print

14. Jago R, Sebire SJ, Davies B, Wood L, Banfield K, Edwards MJ, Powell JE, Montgomery AA, Thompson JL, Fox KR. Increasing children's physical activity through a teachingassistant led extracurricular intervention: process evaluation of the action 3:30 randomised feasibility trial. BMC Public Health. 2015; 15(1): 156.

15. Kasmire KE, Rogers SC, Sturm JJ. Trampoline park and home trampoline injuries. Pediatrics. 2016; 138(3): e20161236

16. Eager D. A jumping revolution. Australasian Parks Leisure. 2015; 18(1): 22.

17. Keytel LR, Goedecke JH, Noakes TD, Hiiloskorpi H, Laukkanen R, van der Merwe L, Lambert EV. Prediction of energy expenditure from heart rate monitoring during submaximal exercise. J Sports Sci. 2005; 23(3): 289-297.

18. Russell JA. A circumplex model of affect. J Pers Soc Psychol. 1980; 39(6): 1161.

19. Hulley A, Bentley N, Clough C, Fishlock A, Morrell F, O'Brien J, Radmore J. Active and passive commuting to school: influences on affect in primary school children. Res Q Exerc Sport. 2008; 79(4):525534.

20. Szakály Z, Bognár J, Barthalos I, Ács P, Ihász F, Fügedi B. Specific heart rate values of 10-12-year-old physical education students during physical activity. J Phys Ed Sport. 2016; 127: 800-805.

21. World Health Organisation. 2018; http://www.who.int/dietphysicalacti vity/physical_activity_intensity/en/

22. Canning KL, Brown RE, Jamnik VK, Salmon A, Ardern CI, Kuk JL. Individuals underestimate moderate and vigorous intensity physical activity. PloS One. 2014; 9(5): e97927.

23. Bendiksen M, Williams CA, Hornstrup $\mathrm{T}$, Clausen $\mathrm{H}$, Kloppenborg J, Shumikhin D, Brito J, Horton J, Barene S, Jackman SR, Krustrup, P. Heart rate response and fitness effects of various types of physical education for 8-to 9-yearold schoolchildren. Eur J Sport Sci. 2014; 14(8): 861-869.

24. Fisher J, Steele J. Questioning the resistance/aerobic training dichotomy: A commentary on physiological adaptations determined by effort rather than exercise modality. J Hum Kinet. 2014; 44: 137-142

25. Tarp J, Child A, White T, Westgate $\mathrm{K}$, Bugge A, Grøntved A, Wedderkopp N, Andersen LB, Cardon G, Davey R, Janz KF, Kriemler S, Northstone K, Page AS, Puder JJ, Reilly JJ, Sardinha LB, van Sluijs EMF, Ekelund U, Wijndaele $\mathrm{K}$, Brage S. Physical activity intensity, bout-duration, and cardiometabolic risk markers in children and adolescents. Int $\mathbf{J}$ Obesity. 2018; Epub ahead of print.

26. Reilly JJ, Jackson DM, Montgomery C, Kelly LA, Slater C, Grant S, Paton JY. Total energy expenditure and physical activity in young Scottish children: mixed longitudinal study. The Lancet. 2004; 363(9404): 211-212.

27. Ainsworth BE, Haskell WL, Leon AS, Jacobs DR, Montoye HJ, Sallis JF, Paffenbarger RS. Compendium of physical activities. Med Sci Sports Exerc. 1993; 25(1): 71-80.

28. Harrell JS, McMurray RG, Baggett CD, Pennell ML, Pearce PF, Bangdiwala SI. Energy costs of physical activities in children and adolescents. Med Sci Sports Exerc. 2005; 37(2): 329-336

29. Butte NF, Watson KB, Ridley K, Zakeri IF, McMurray RG, Pfeiffer 
KA, Crouter SE, Hermann SD, Bassett DR, Long A, Berhane Z, Trost SG, Ainsworth BE, Berrigan D, Fulton JE. A youth compendium of physical activities: Activity codes and metabolic intensities. Med Sci Sports Exerc. 2018; 50(2): 246-256

30. Allender S, Cowburn G, Foster C. Understanding participation in sport and physical activity among children and adults: a review of qualitative studies. Health Educ Res. 2006; 21(6): 826-835.

31. Hesketh KR, Lakshman R, van Sluijs EMF. Barriers and facilitators to young children's physical activity and sedentary behaviour: a systematic review and synthesis of qualitative literature. Obes Rev. 2017; 18(9): 987-1017

32. Reinberg A, Reinberg O, Mechkouri M, Touitou Y, Smolensky MH. Daily, weekly, and annual patterns in children's accidental sport injuries. Chronobiology Int. 2018; 35(5): 597-616

33. Nysted M, Drogset JO. Trampoline injuries. Br J Sports Med. 2006; 40(12), 984-987

34. Atkinson G, Batterham AM. True and false interindividual differences in the physiological response to an intervention. Exp Physiol. 2015; 100(6): 577-588 